\title{
ELECTRICAL AND MAGNETIC PROPERTIES
}

\section{Magnetic Behavior of the Nanophase of $\mathrm{YbNi}_{2}$ Alloys}

\author{
V. A. Ivanshin ${ }^{a, *}$, E. M. Gataullin ${ }^{a}$, A. A. Sukhanov ${ }^{b}$, N. A. Ivanshin ${ }^{c}$, \\ D. P. Rojas ${ }^{d}$, and L. Fernández Barquín ${ }^{e}$ \\ ${ }^{a}$ Institute of Physics, Kazan Federal (Volga region) University, ul. Kremlevskaya 18, Kazan, 420008 Russia \\ ${ }^{b}$ Zavoiskii Kazan Physicotechnical Institute, Kazan Research Center, Russian Academy of Sciences, \\ Sibirskii tract 10/7, Kazan, 420029 Russia \\ ${ }^{c}$ Kazan State University of Architecture and Engineering, ul. Zelenaya 1, Kazan, 420043 Russia \\ ${ }^{d}$ Departamento de Estructuras y Física de Edificación-ETSAM, Universidad Politécnica de Madrid, \\ Av. Juan Herrera, 4, Madrid 28040, Spain \\ ${ }^{e}$ DCITIMAC, Facultad de Ciencias, Universidad de Cantabria, Av. de los Castros s/n, Santander 39005, Spain \\ *e-mail: Vladimir.Ivanshin@kpfu.ru \\ Received June 2, 2016; in final form, November 2, 2016
}

\begin{abstract}
Variations in magnetic properties of the heavy-fermion $\mathrm{YbNi}_{2}$ alloy when milled in a high energy ball milling system have been investigated. The ferromagnetic transition $\left(T_{\mathrm{C}}=10.4 \mathrm{~K}\right)$ in the initial sample almost vanishes after milling, which leads to the appearance of a magnetic transition at $T^{*}=3.2 \mathrm{~K}$ in nanocrystallites. Before milling, processes of spin-lattice relaxation of the Orbach-Aminov type with the participation of the first excited Stark sublevel of the $\mathrm{Yb}^{3+}$ ion located at $75 \mathrm{~K}$ are dominating in the electron spin dynamics in the paramagnetic phase of the alloy. A comparative study of the temperature dependence of the magnetic properties and spectra of electron paramagnetic resonance in poly- and nanocrystalline samples indicates the existence of a magnetic inhomogeneity of the compound arising upon milling.
\end{abstract}

Keywords: heavy fermions, magnetic susceptibility, electron paramagnetic resonance, nanocrystallites

DOI: $10.1134 / \mathrm{S} 0031918 X 17040044$

\section{INTRODUCTION}

In the last decade, much attention was paid to investigating the physical behavior of strongly correlated systems in the nanophase state. This is explained by their existence in unusual physical effects, such as the intermediate valence, non-Fermiliquid behavior, and quantum critical points. In contrast to the cerium-based compounds, ytterbiumbased systems have been studied insufficiently, apparently because of the difficulties related to the high vapor pressure of ytterbium during the synthesis of its compounds. However, the results of numerous experiments show that these materials deserve special attention, since they can manifest physical properties capable of explaining the nature of many new phenomena. For example, the first investigation of the size effects on the properties of ytterbium alloys performed in 2008 [1] showed that a decrease in the size of the particles leads to a sharp increase in the amount of divalent ytterbium atoms on the surface of the sample compared to its bulk and, thus, leads to a change in the general electronic state of the system.

Among the most informative methods of studying local characteristics, magnetic structure, and its disordering, as well as surface effects, are radiospectro- scopic methods, such as nuclear magnetic resonance (NMR) and electron paramagnetic resonance (EPR). These methods make it possible to efficiently describe both the paramagnetic (PM) and ferromagnetic (FM) phases in crystalline samples and nanomaterials [2-4]. Recently, we have shown that the Laves phase $\mathrm{YbNi}_{2}$ represents an unique example of FM compounds with clearly pronounced heavy-fermion (HF) behavior and refers to concentrated Yb-based Kondo lattices that exhibit the existence of an EPR effect without the introduction of special PM ions as EPR probes [5]. In this work, we report on measurements of the magnetic susceptibility and EPR spectra in $\mathrm{YbNi}_{2}$ powders prepared by mechanical milling. Thus, for the first time, we used the EPR method to study size effects in a $\mathrm{HF}$ Yb-based alloy containing no doping impurities.

\section{EXPERIMENTAL}

The initial alloy was obtained by the method of arc melting using stoichiometric amounts of pure elements of $\mathrm{Yb}$ (4N-Alfa) and Ni (5N-Alfa) in an arc furnace under an Ar protective atmosphere. Then, $2 \mathrm{~g}$ of the synthesized alloy were crushed and milled in a Retsch PM 400/2 planetary ball milling system at a rotation speed of $200 \mathrm{rpm}$ using milling balls and con- 


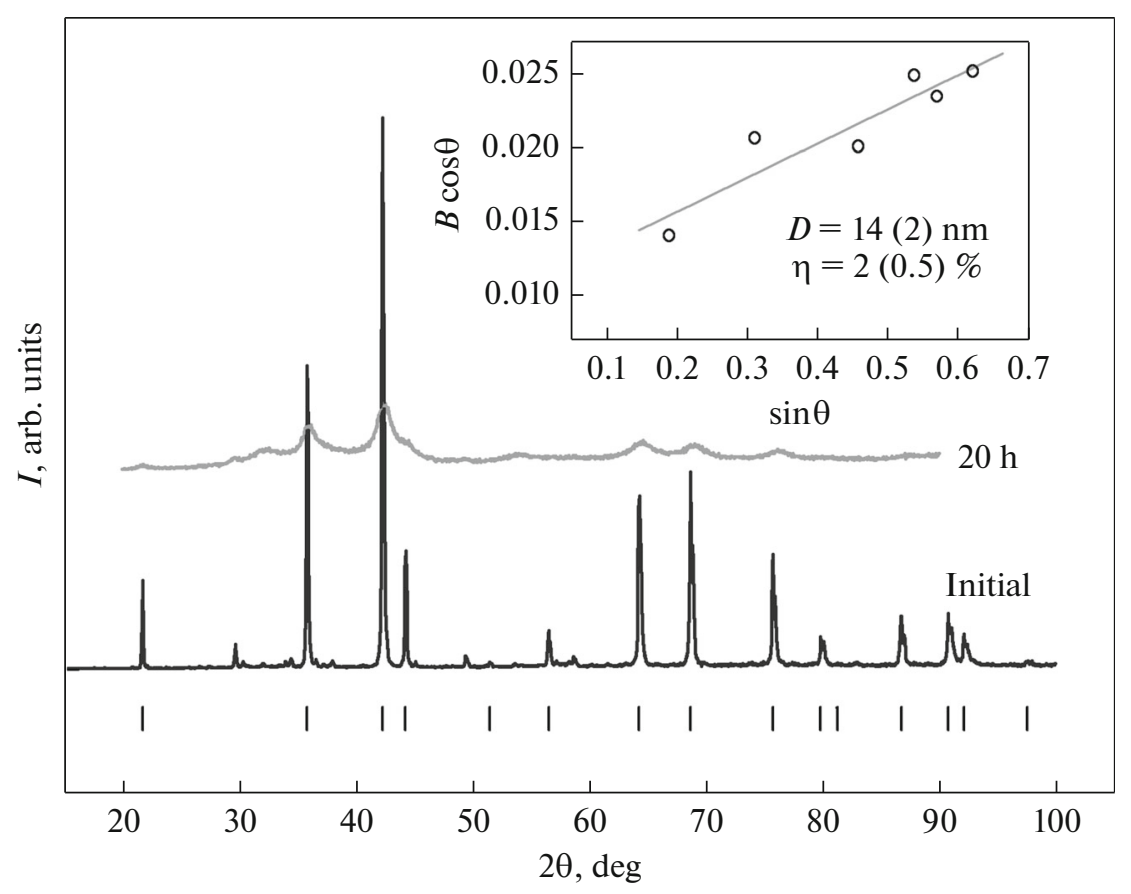

Fig. 1. Experimental $\mathrm{X}$-ray diffraction spectra of the $\mathrm{YbNi}_{2}$ powder before and after milling for $20 \mathrm{~h}$. Vertical markers indicate the positions of the Bragg peaks corresponding to the cubic structure (space group $F d 3 m$ ). Inset shows a Williamson-Hall plot for the $\mathrm{YbNi}_{2}$ alloy milled for $20 \mathrm{~h}$.

tainers made of tungsten carbide. Small amounts of the material were removed after milling for $20 \mathrm{~h}$ upon the unaltered ratio of the masses of the balls and the initial material equal to $12: 1$. To prevent the oxidation of the obtained samples, they were treated and stored in a pure chamber under a protective Ar atmosphere.

The measurements of the magnetic susceptibility were carried out using a Quantum Design PPMS setup. The X-ray diffraction (XRD) analysis was performed using a Bruker diffractometer in $\mathrm{Cu} K \alpha$ radiation. The EPR spectra were recorded on an EMX plus (Bruker BioSpin Gmbh, Germany) continuous-wave $\mathrm{X}$-band spectrometer $(v \sim 9.4 \mathrm{GHz})$ using an ESR900 Oxford cryogenics cryostat that operates in a temperature range of 4.5-290 K with a temperature stability of about $0.1 \mathrm{~K}$.

\section{RESULTS AND DISCUSSION}

Figure 1 shows XRD spectra of $\mathrm{YbNi}_{2}$ powders before and after milling for $20 \mathrm{~h}$. The milled sample exhibits a broadening of the peaks and a decrease in their intensity as compared to the initial alloy that are similar to those observed earlier for other intermetallic compounds [6-8]. All the reflections were identified as corresponding to the Laves phase belonging to the crystallographic space group $F d 3 m$. As was already reported in [8], in the initial sample there were observed small $(<4 \%)$ amounts of the orthorhombic $\mathrm{YbNi}$. In the milled sample, there appeared also a peak near $2 \theta=28^{\circ}$ corresponding to $\mathrm{Yb}_{2} \mathrm{O}_{3}$.
Nevertheless, we assume that the magnetic contributions from the impurity phase of $\mathrm{YbNi}$ (which is paramagnetic up to $1.4 \mathrm{~K})$ and from $\mathrm{Yb}_{2} \mathrm{O}_{3}\left(T_{\mathrm{N}}=\right.$ $2.1 \mathrm{~K})$ are negligibly small on the order of magnitude $\left(\sim 10^{-3}\right)$ compared to the contribution from the main component $\mathrm{YbNi}_{2}$. Also, as it was found earlier [1] for other $\mathrm{Yb}$-based alloys, the process of milling favors the nonmagnetic state rather than a strengthening of magnetism.

The size of particles and the magnitude of microdeformations were estimated by the Williamson-Hall method, which is assumed to be most suitable for milled alloys [1]. According to this procedure [9], it is assumed that the broadened $B_{h k l}$ line is the Bragg reflection $(h k l)$ from fine crystallites with a size that corresponds to the Scherrer equation $B_{h k l}=$ $(K \lambda / D) / \cos \theta_{h k l}$. Here, $K$ is the form factor, $\lambda$ is the wavelength of the X-ray diffraction radiation, $\theta_{h k l}$ is the Bragg angle, and $D$ is the crystallite size. The plotting of the dependence of $B_{h k l} \cos \theta_{h k l}$ on $\sin \theta_{h k l}$ makes it possible to estimate the magnitude of microdeformations $(\eta)$ from the slope of the line and the crystallite size from the intersection of this line with the vertical axis. Using this method for the XRD data on the $\mathrm{YbNi}_{2}$ alloy milled for $20 \mathrm{~h}$, we obtained results shown in the inset in Fig. 1 and the values of $D=14(2) \mathrm{nm}$ and $\eta=2.0(0.5) \%$.

Figure 2 shows the data on the measurements of the magnetic susceptibility in a dc magnetic field $H=$ $100 \mathrm{Oe}$ for the initial $\mathrm{YbNi}_{2}$ alloy and the alloy milled 


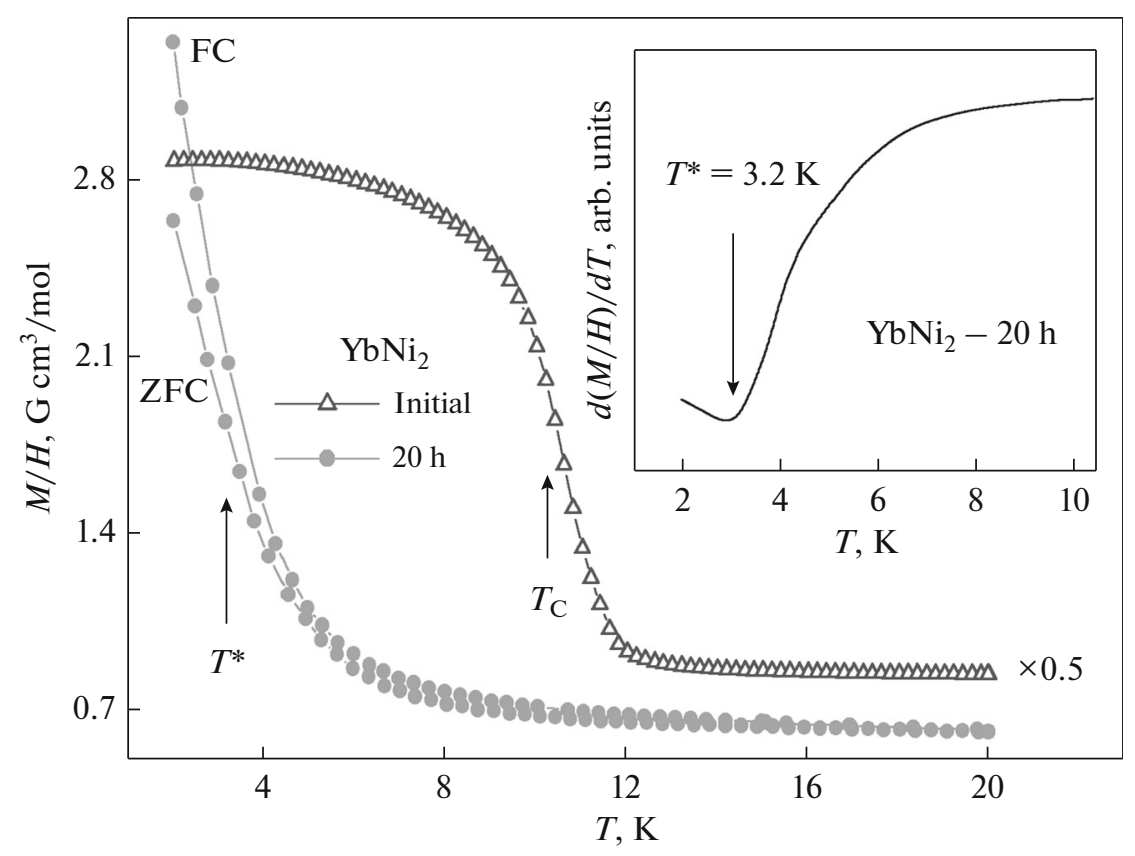

Fig. 2. Temperature dependence of the magnetic susceptibility in a dc regimes magnetic field $H=100 \mathrm{Oe}$ for the initial $\mathrm{YbNi} \mathrm{H}_{2}$ alloy and for the milled sample in zero field cooled (ZFC) and field cooled (FC) regimes. Inset shows the temperature dependence of the first derivative of the magnetic susceptibility for the milled sample.

for $20 \mathrm{~h}$. For clearness, the data for the initial sample were divided by two. As was established earlier, in the polycrystalline sample there was revealed an FM transition at $T_{\mathrm{C}}=10.4 \mathrm{~K}$ [8]. It is interesting that in the milled sample, no signs of this transition were found. On the contrary, as is shown in the inset in Fig. 2, the displacement of the minimum of the first derivative to the region of lower temperatures indicated the existence of a magnetic transition at $T^{*}=3.2 \mathrm{~K}$. The probability of this transition is also confirmed by the irreversibility of the curves obtained for the milled sample in zero field cooled (ZFC) and field cooled (FC) regimes, and in the temperature range below $4 \mathrm{~K}$. This means that, on the whole, this behavior coincides with the previously revealed effects for poly- and nanocrystalline $\mathrm{TbAl}_{2}$ alloys, in which the process of milling led to the complete disappearance of the FM transition at $T_{\mathrm{C}}=$ $105 \mathrm{~K}$ and to the appearance of a magnetic transition at a lower temperature $\left(T_{\mathrm{f}}=45 \mathrm{~K}\right)[6]$.

The magnetic transition in the case of milled $\mathrm{YbNi}_{2}$ alloys at $T^{*}=3.2 \mathrm{~K}$ is probably also caused by analogous factors that cause the spin disorder as a result of the milling of the alloy. This result is by no means unexpected if we take into account the fact that a decrease in the size of particles to $14 \mathrm{~nm}$ leads to a sharp increase of the surface/volume ratio, which indeed leads to an increase in the spin disorder on the surface of the particles. Therefore, we think to perform a further investigation of the nature of this transition. Here, measurements of the magnetic susceptibility in magnetic fields of various strengths, as well as study of the magnetic susceptibility in ac magnetic fields can be most suitable. However, a careful analysis of the nature of the possible magnetic transition at $T^{*}$ will be considered elsewhere.

The EPR spectra for the initial $\mathrm{YbNi}_{2}$ sample and the sample after milling for $20 \mathrm{~h}$ for three selected temperatures, namely, $4.2,15$, and $30 \mathrm{~K}$ located below and above $T_{\mathrm{C}}$, are shown in Fig. 3 .

As a rule, the EPR spectra are described based on the measurements of two parameters, i.e., the effective $g$ factor $g=h v /\left(\mu_{\mathrm{B}} H_{\text {res }}\right)$, where $H_{\text {res }}$ is the value of the resonance magnetic field, and the EPR linewidth $\Delta H_{\mathrm{pp}}$. Upon passing through $T_{\mathrm{C}}$, the EPR signals markedly change their positions, linewidth, and shape. In the PM state of the initial sample between 11 and $18 \mathrm{~K}$, an asymmetric Dyson EPR line was observed with $g \sim 2.34$ and $\Delta H_{\mathrm{pp}} \sim 800$ Oe at $15 \mathrm{~K}$ (Fig. 3b). The behavior of the linewidth proved to be typical of a paramagnet in the vicinity of $T_{\mathrm{C}}[3,10]$. As the temperature increases from the minimum value, the magnitude of $\Delta H_{\mathrm{pp}}$ decreases and reaches a minimum near $T_{\mathrm{C}}$, then increases exponentially upon a further increase in temperature. Recently, we have explained the temperature dependence of $\Delta H_{\mathrm{pp}}$ in the PM phase of $\mathrm{YbNi}_{2}$ by the effect of the contribution from spin-lattice interactions due to the process of spin-lattice relaxation of the Orbach-Aminov type through the first excited Kramers doublet of the $\mathrm{Yb}^{3+}$ ion in a cubic crystal field, which is located at $75 \pm 5 \mathrm{~K}$ [5]. According to some theories [11-13], this type of 
(a)

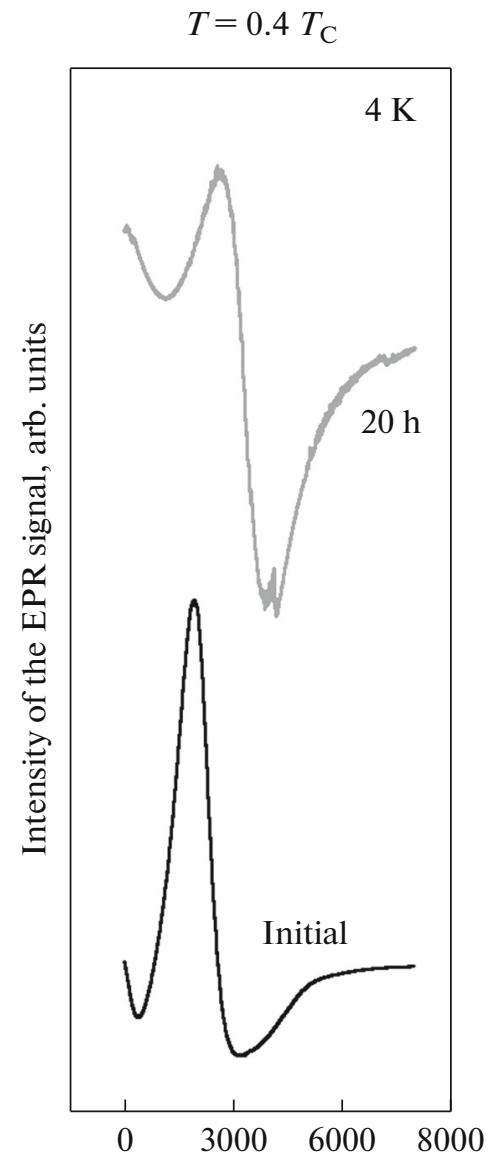

(b)

$T=1.43 T_{\mathrm{C}}$

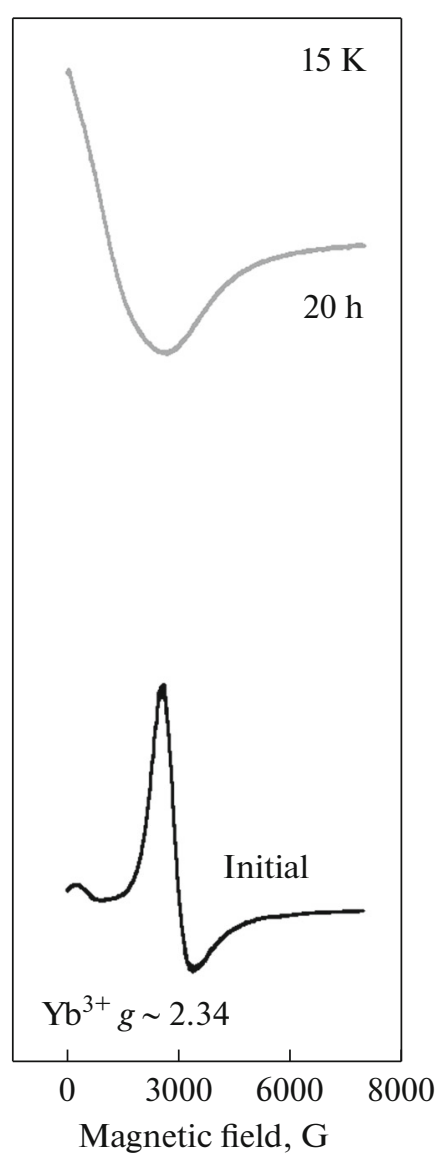

(c)

$T=2.86 T_{\mathrm{C}}$

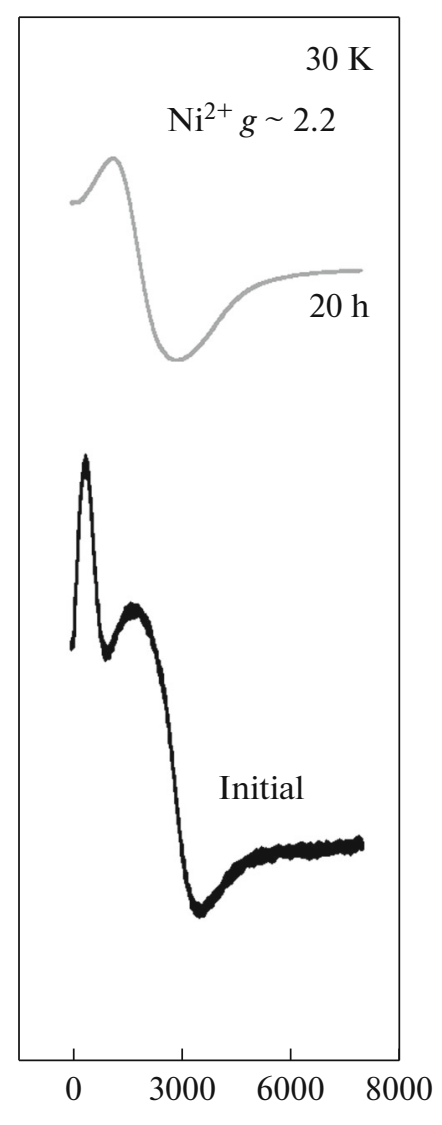

Fig. 3. EPR spectra of the initial and milled (for $20 \mathrm{~h}$ ) samples of the $\mathrm{YbNi}_{2}$ alloy for three characteristic values of the temperature below and above $T_{\mathrm{C}}$ : (a) 4 , (b) 15 , and (c) $30 \mathrm{~K}$.

EPR absorption in concentrated HF materials is due to the effective hybridization between the localized $f$ electrons and conduction electrons in the presence of FM short-range-order correlations, which lead to an increase in the susceptibility and the appearance of an EPR signal.

Contrary to this, in the milled sample, the EPR lines are shifted significantly at $T<T_{\mathrm{C}}$ (by about $150-$ $200 \mathrm{Oe}$ ) from their positions in the initial alloy into the range of stronger fields and, for the temperature range of $11<T<18 \mathrm{~K}$, in the opposite direction, which indicates the existence of a significant magnetic inhomogeneity and of a spin disorder that arise upon milling (Figs. 3a, 3b). Finally, at $30 \mathrm{~K}$ (Fig. 3c), the values of the EPR linewidth, just like the resonance fields, become almost identical in the initial and milled samples. Values of the $g$ factors of about 2.2 measured for this symmetrical absorption signal correlate well with the literature data on the ferromagnetic resonance in metallic nickel [14]. It is found that the rare-earth atoms with their unstable $f$ electron shell exhibit a lower valence at the surface only, rather than in the bulk of the sample [15]. Therefore, we could assume that, simultaneously with a decrease in the size of particles an increase in the number of $\mathrm{Yb}^{2+}$ atoms on the surface, and a decrease in the number of $\mathrm{Yb}^{3+}$ atoms occur as was already found in the case of $\mathrm{YbAl}_{3}$ [1]. Thus, the mechanical milling process destroys the FM state in bulk $\mathrm{YbNi}_{2}$ alloy and leads to the growth of a magnetic phase at lower temperatures.

\section{CONCLUSIONS}

In this work, we obtained poly- and nanocrystalline samples of $\mathrm{YbNi}_{2}$. An analysis of XRD data made it possible to estimate a decrease in the size of particles to $14 \mathrm{~nm}$ after milling for $20 \mathrm{~h}$. The results of the measurements of the EPR and magnetic susceptibility indicate that the FM transition that exists in the initial alloy evolves into a spin disorder state in the nanosized sample with the appearance of a magnetic transition at $T^{*}=3.2 \mathrm{~K}$. The study of this transition requires additional investigations. 


\section{ACKNOWLEDGMENTS}

This work was supported by a subsidy allocated to the Kazan Federal University for the state assignment in the sphere of scientific activities. D.P. Rojas and L. Fernández-Barquín were supported by the Government of the Spain (grant MAT 2014-55049-C2-R).

\section{REFERENCES}

1. D. P. Rojas, L. Fernández Barquín, J. I. Espeso, J. Rodríguez Fernández, and J. Chaboy, "Reduction of the $\mathrm{Yb}$ valence in $\mathrm{YbAl}_{3}$ nanoparticles," Phys. Rev. B: Condens. Matter Mater. Phys. 78, 094412 (2008).

2. A. V. Chadwick, I. J. F. Poplett, D. T. S. Maitland, and M. E. Smith, "Oxygen speciation in nanophase $\mathrm{MgO}$ from solid-state ${ }^{17} \mathrm{O}$ NMR," Chem. Mater. 10, 864870 (1998).

3. G. Singh and S. V. Bhat, "Effect of size reduction on magnetic ordering in $\mathrm{Bi}_{0.2} \mathrm{Sr}_{0.8} \mathrm{MnO}_{3}$," J. Appl. Phys. 115, 17E130 (2014).

4. K. Baberschke, "From local moment EPR in superconductors to nanoscale ferromagnets," J. Supercond. Nov. Magn. 19, 77-84 (2006).

5. E. M. Gataullin, V. A. Ivanshin, D. P. Rojas, and L. Fernández-Barquin, "Electron spin resonance of the ferromagnetic $\mathrm{YbNi}_{2}$ alloy," JPS Conf. Proc. 3, 012028 (2014).

6. D. P. Rojas, L. Fernández Barquín, J. Rodríguez Fernández, J. I. Espeso, and J. C. Gómez Sal, "Size effects in the magnetic behaviour of $\mathrm{TbAl}_{2}$ milled alloys," J. Phys.: Condens. Matter 19, 186214 (2007).

7. D. P. Rojas, L. Fernández Barquín, J. Rodríguez Fernández, J. I. Espeso, and J. C. Gómez Sal, "Magnetization and specific heat of nanocrystalline rare- earth $\mathrm{TbAl}_{2}, \mathrm{TbCu}_{2}$ and $\mathrm{GdAl}_{2}$ alloys," J. Phys.: Conf. Ser. 200, 072080 (2010).

8. D. P. Rojas, L. Fernández Barquín, C. EchevarriaBonet, and J. Rodríguez Fernández," "YbNi 2 : A heavy fermion ferromagnet," Solid State Commun. 152, 1834-1837 (2012).

9. G. K. Williamson and W. H. Hall, "X-ray line broadening from filed aluminum and wolfram," Acta Metall. 1, 22-31 (1953).

10. V. A. Ivanshin, J. Deisenhofer, H.-A. Krug von Nidda, A. Loidl, A. A. Mukhin, A. M. Balbashov, and M. V. Eremin, "ESR study in lightly doped $\mathrm{La}_{(1-x)} \mathrm{Sr}_{x} \mathrm{MnO}_{3}$," Phys. Rev. B: Condens. Matter Mater. Phys. 61, 6213-6219 (2000).

11. P. Schlottmann, "Electron spin resonance in heavyfermion systems," Phys. Rev. B: Condens. Matter Mater. Phys. 79, 045104 (2009).

12. E. Abrahams and P. Wölfle, "Electron spin resonance in Kondo systems," Phys. Rev. B: Condens. Matter Mater. Phys. 78, 104423 (2008).

13. A. Ramirez and P. Coleman, "Theory of the electron spin resonance in the heavy fermion metal $\beta-\mathrm{YbAlB}_{4}$," Phys. Rev. Lett. 112, 116405 (2014).

14. D. S. Rodbell, "Ferromagnetic resonance absorption linewidth of nickel metal. Evidence for Landau-Lifshitz damping,” Phys. Rev. Lett. 13, 471-474 (1964).

15. M. Domke, C. Laubschat, E. V. Sampathkumaran, M. Prietsch, T. Mandel, G. Kaind, and H. U. Middelmann, "Bulk and surface valence in $\mathrm{YbPd}_{\mathrm{x}}$ compounds," Phys. Rev. B: Condens. Matter 32, 80028006 (1985). 